\title{
Closed-Loop Carrier Phase Synchronization Techniques Motivated by Likelihood Functions
}

\author{
H. Tsou and S. Hinedi \\ Communications Systems Research Section \\ M. Simon \\ Telecommunications Systems Section
}

\begin{abstract}
This article reexamines the notion of closed-loop carrier phase synchronization motivated by the theory of maximum a posteriori phase estimation with emphasis on the development of new structures based on both maximum-likelihood and averagelikelihood functions. The criterion of performance used for comparison of all the closed-loop structures discussed is the mean-squared phase error for a fixed-loop bandwidth.
\end{abstract}

\section{Introduction}

It is well known [1] that estimation of an unknown parameter based on a likelihood function approach is optimum in the sense of maximizing the a posteriori probability of the parameter given the observation. For the case where the unknown parameter is the random phase of a carrier received in a background of additive white Gaussian noise (AWGN), optimum open-loop structures have been derived for implementing the resulting phase estimate $[2,3]$. Herein, these structures are referred to as "open-loop carrier phase estimators."

When the carrier is data modulated, the conditional probability density function (pdf) of the observation-given the carrier phase-depends on the data sequence that exists during the interval of observation for the received signal. Hence, before maximizing this function with respect to the carrier phase, one has to choose how to eliminate its dependence on the unknown data sequence. If one is interested in determining only the optimum carrier phase estimate, the appropriate choice is to average the conditional pdf over the unknown data sequence. We shall refer to the phase estimate obtained by this process as the "average-likelihood" (AL) estimate. If, however, one is interested in joint phase estimation and data detection, the appropriate choice is to first maximize the conditional pdf with respect to the data sequence (resulting in the most probable sequence), and then to maximize it with respect to the carrier phase. ${ }^{1}$ We shall refer to the phase estimate obtained by this process as the "maximum-likelihood"

\footnotetext{
${ }^{1}$ In principle, the order of maximization operations could be reversed.
} 
(ML) estimate. ${ }^{2}$ It has often been conjectured, although never proven, that from the standpoint of phase estimation alone, the ML phase estimate is suboptimum to the AL estimate. Because of this, what is typically done in practice is to derive the $\mathrm{AL}$ carrier phase estimate and then use this estimate as the phase of a demodulation reference signal for performing bit-by-bit data detection. However, it should be understood that, from the standpoint of joint estimation of data and carrier phase, this sequential operation of first deriving the carrier phase estimate in the absence of any knowledge of the data (the $\mathrm{AL}$ approach) and then detecting the ensuing data using the phase estimate so derived is, in general, suboptimum.

Aside from the optimality of the AL and ML approaches to open-loop estimation of carrier phase, likelihood functions have also been used as motivation for closed-loop carrier phase synchronization. Emphasis is placed on the word "motivation" since, indeed, there is no guarantee that the resulting closed-loop schemes are optimal; nor can one guarantee that those schemes motivated by the AL approach will outperform those motivated by the ML approach (although typically this turns out to be the case, as we shall show.) Nonetheless, as we shall see, closed-loop carrier phase estimation schemes motivated by likelihood functions do indeed yield good tracking performance (as measured by the mean-squared value of the loop phase error). In fact, under suitable assumptions, many of them are synonymous with well-known carrier tracking loops, e.g., the I-Q Costas loop and the I-Q decision feedback or polarity-type Costás loop $[4,5]$ that have been around for many decades.

It is the intent of this article to explore in more detail the structure and performance of closed-loop carrier phase synchronization loops motivated by likelihood functions, i.e., those in which the derivative (or some monotonic function of the derivative) of the conditional pdf of the observation given the carrier phase is used as an error signal in a closed-loop phase estimation scheme. Herein, for the purpose of abbreviated notation, we shall refer to such loops as AL and ML closed loops depending on the particular likelihood function used to define the error signal.

It is important at this point to mention that the notion of closed loops based on likelihood functions according to the above definition is indeed not new, and one should not attribute its originality to the authors of this article. Rather, the purpose of this article is to expand upon this notion and present some new loops motivated by likelihood functions along with their tracking performances. As such, we are not reinventing the wheel but, rather, adding some more spokes to it. Our specific motivation for reexamining this problem comes from a deep-space communication application involving the Galileo S-band $(2.3 \mathrm{GHz})$ mission, which employs low-rate $(r=1 / 4)$ concatenated Reed-Solomon/convolutionally encoded binary phase-shift keying (BPSK) [6]. Because of a malfunctioning high-gain X-band (8.4 GHz) antenna, the mission must rely on a low-gain S-band antenna (and, thus, much reduced link margin) for data transmission back to Earth. At Jupiter encounter, the symbol energy-to-noise spectral density ratio, $E_{s} / N_{0}$, could be as low as $-11 \mathrm{~dB}$. One technique for improving this situation is to use antenna array combining [7] wherein the signals from multiple antennas, either collocated or at distant geographical locations, are combined to build $E_{s} / N_{0}$. Even then the equivalent $E_{s} / N_{0}$ could still be as low as $-5 \mathrm{~dB}$. Thus, in our application, there is a serious need to find as efficient a carrier tracking loop as possible in the sense of producing minimum phase jitter at very low $E_{s} / N_{0}$. In the more general context, it is important to point out that, in coded systems, the carrier-loop performance is dependent on the symbol energy-to-noise ratio $E_{s} / N_{0}$ rather than the bit energy-to-noise ratio $E_{b} / N_{0}$ and, thus, becomes critical when $E_{s} / N_{0}$ becomes small, despite the fact that $E_{b} / N_{0}$ might be large. In uncoded systems where $E_{s} / N_{0}=E_{b} / N_{0}$ and is large, the search for a more efficient carrier tracking loop is somewhat academic since the known configurations perform quite well and are virtually identical to one another.

\footnotetext{
${ }^{2}$ In the strictest of parlance, both the $\mathrm{AL}$ and the ML phase estimates are maximum-likelihood estimates since the term "maximum-likelihood estimation" is typically reserved for estimating a purely unknown (uniformly distributed) random parameter. However, to allow for distinguishing between the two different ways in which the data sequence is handled, we shall use the above terminology.
} 
The hierarchical structure of the problem and also the way in which it is addressed in this article is illustrated by the tree diagram of Fig. 1. We have already discussed the first level of the overall dichotomy in terms of the ML and AL approaches. This level of the chart as well as those below it will take on more meaning as soon as we develop a mathematical formulation of the problem in Section III.

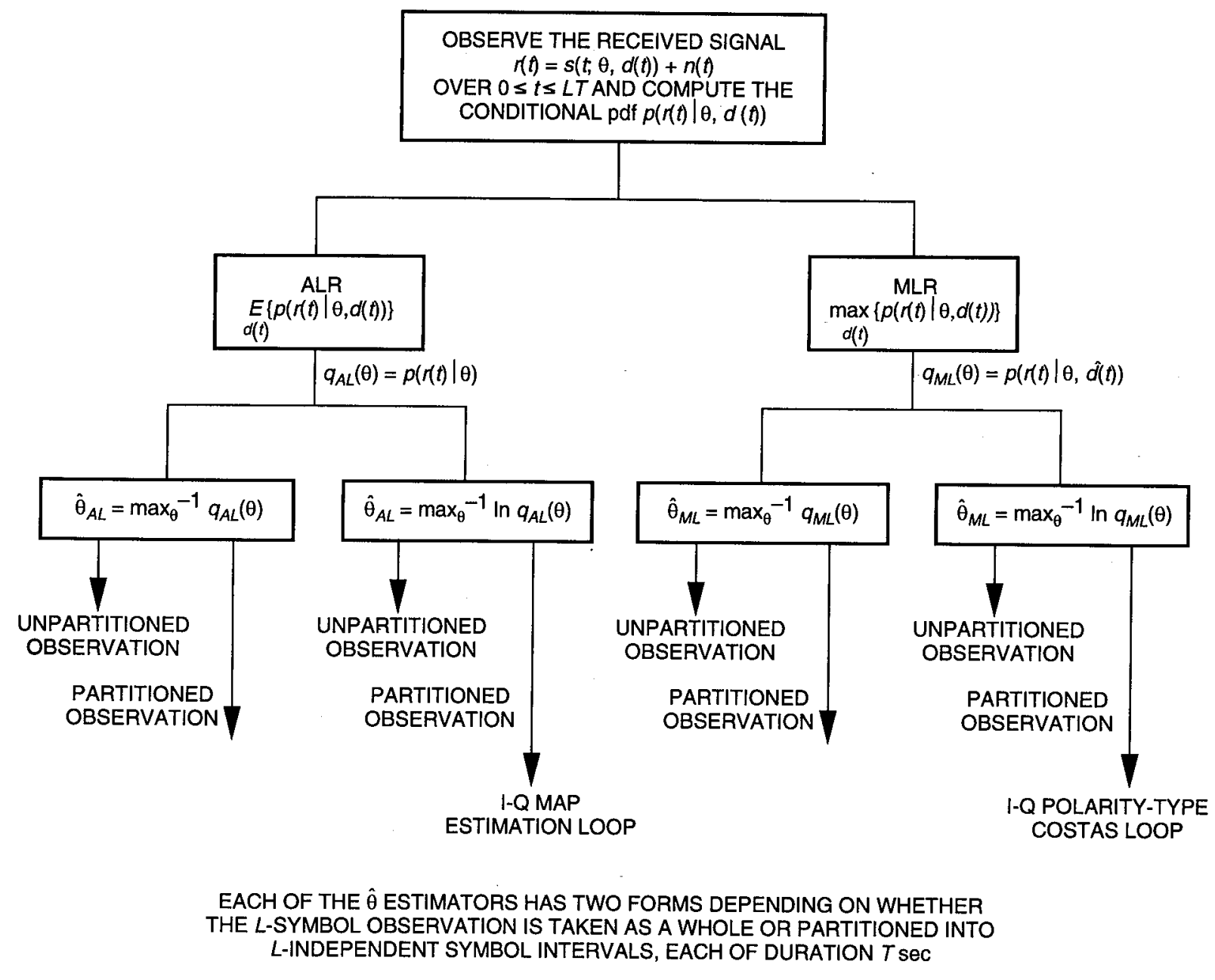

Fig. 1. A hierarchical structure of the open-/closed-loop carrier phase estimation problem for data-modulated signals.

\section{System Model}

Consider a system that transmits BPSK ${ }^{3}$ modulation over an AWGN channel. As such, the received signal takes the form

$$
r(t)=\sqrt{2 S} d(t) \sin \left(\omega_{c} t+\theta\right)+n(t)=s(t ; \theta, d(t))+n(t)
$$

where $S$ denotes the received power, $\omega_{c}$ is the carrier frequency in $\mathrm{rad} / \mathrm{sec}, \theta$ is the unknown phase assumed to be uniformly distributed in the interval $(-\pi, \pi), n(t)$ is an AWGN with single-sided power

\footnotetext{
${ }^{3}$ We restrict ourselves to the case of binary modulation. By a straightforward extension of the procedures discussed, the results can easily be extended to $M$-ary modulation.
} 
spectral density $N_{0} \mathrm{~W} / \mathrm{Hz}$, and $d(t)$ is a binary-valued $( \pm 1)$ random pulse train defined by the rate $1 / T$ binary data sequence $\left\{d_{i}\right\}$ and the rectangular pulse shape, $p(t)$, as

$$
d(t)=\sum_{i=-\infty}^{\infty} d_{i} p(t-i T), \quad p(t)= \begin{cases}1 ; & 0 \leq t \leq T \\ 0 ; & \text { otherwise }\end{cases}
$$

For an observation interval of $L$ bits [we assume without loss of generality the interval $(0, L T)]$, the conditional pdf of the received signal (observation) given the unknown phase and the particular data sequence, $d_{i}$, transmitted in that interval is easily shown to be

$$
p\left(r(t) \mid \theta, d_{i}(t)\right)=C_{0} \exp \left(\frac{2 \sqrt{2 S}}{N_{0}} \int_{0}^{L T} r(t) d_{i}(t) \sin \left(\omega_{c} t+\theta\right) d t\right) \triangleq q_{i}(\theta)
$$

where $d_{i}(t)$ is the transmitted waveform corresponding to the transmitted sequence in accordance with Eq. (2) and $C_{0}$ is a constant of proportionality. To proceed further, we must now choose between AL and ML approaches.

\section{Closed Loops Motivated by the AL Approach}

\section{A. Structures}

Suppose that we are interested in estimating only the carrier phase, $\theta$. Then, as previously mentioned, the appropriate approach is to average $p\left(r(t) \mid \theta, d_{i}(t)\right)$ over all possible $\left(2^{L}\right)$ and equally likely data sequences yielding the conditional pdf $p(r(t) \mid \theta) \triangleq q_{A L}(\theta)$. One AL open-loop phase estimate (herein referred to as "AL open-loop estimator no. 1") is obtained by finding the value of $\theta$ that maximizes $q_{A L}(\theta)$, namely (see Fig. 1: $\hat{\theta}_{A L} \triangleq \max _{\theta}^{-1} q_{A L}(\theta)$, unpartitioned observation)

$$
\hat{\theta}_{A L_{1}} \triangleq \max _{\theta}{ }^{-1} \sum_{i=1}^{2^{L}} \exp \left(\frac{2 \sqrt{2 S}}{N_{0}} \int_{0}^{L T} r(t) d_{i} \sin \left(\omega_{c} t+\theta\right) d t\right)
$$

where the inverse maximum notation " $\max ^{-1} f(\theta)$ " denotes the value of $\theta$ that maximizes $f(\theta)$. Alternately, breaking up the integration over the entire observation into a sum of integrals on each bit interval and recognizing that the data bits are independent, identically distributed (iid) binary random variables, then $p(r(t) \mid \theta)$ can be expressed as a product of hyperbolic cosine functions. A second AL open-loop phase estimate (herein referred to as "AL open-loop estimator no. 2") is obtained by finding the value of $\theta$ that maximizes this product form of $q_{A L}(\theta)$, which corresponds to partitioning the observation into its individual bit intervals. The result is (see Fig. 1: $\hat{\theta}_{A L} \triangleq \max _{\theta}^{-1} q_{A L}(\theta)$, partitioned observation)

$$
\hat{\theta}_{A L_{2}} \triangleq \max _{\theta}{ }^{-1} \prod_{k=0}^{L-1} \cosh \left(\frac{2 \sqrt{2 S}}{N_{0}} \int_{k T}^{(k+1) T} r(t) \sin \left(\omega_{c} t+\theta\right) d t\right)
$$

It is important to emphasize here (and we shall repeat this emphasis later on in the closed-loop discussion) that partitioning or not partitioning the observation interval has no effect on the value of the optimum estimator nor on its performance. That is, optimum open-loop $\hat{\theta}_{A L_{1}}$ and $\hat{\theta}_{A L_{2}}$ are mathematically identical. The difference between the two lies solely in their implementation and likewise the difference in the closed-loop implementations motivated by these estimates, as we shall see shortly. 
Finally, one could obtain an AL open-loop estimator by maximizing any monotonic function of $q_{A L}(\theta)$, for example $\ln q_{A L}(\theta)$. The reason for choosing the natural logarithm as the monotonic function is to simplify the mathematics, i.e., to convert the $L$-fold product in Eq. (5) to an $L$-fold sum. Thus, the third AL open-loop phase estimate (herein referred to as "AL open-loop estimator no. 3 ") is obtained by finding the value of $\theta$ that maximizes $\ln q_{A L}(\theta)$ with $q_{A L}(\theta)$ in its partitioned form. The result is (see Fig. 1: $\hat{\theta}_{A L} \triangleq \max _{\theta}^{-1} \ln q_{A L}(\theta)$, partitioned observation)

$$
\hat{\theta}_{A L_{3}} \triangleq \max _{\theta}{ }^{-1} \sum_{k=0}^{L-1} \ln \cosh \left(\frac{2 \sqrt{2 S}}{N_{o}} \int_{k T}^{(k+1) T} r(t) \sin \left(\omega_{c} t+\theta\right) d t\right)
$$

Block diagram implementations of AL open-loop estimator no. 1 [Eq. (4)] and AL open-loop estimator no. 3 [Eq. (6)] are illustrated in Fig. 2, no. 3 being the form most commonly found in discussions of open-loop maximum a posteriori (MAP) carrier phase estimation. In drawing these implementations, we have quantized the unknown phase into $Q$ values, and thus the maximization over the continuous phase parameter $\theta$ in Eqs. (4) and (6) is approximated by maximization over a $Q$-quantized version of this parameter.
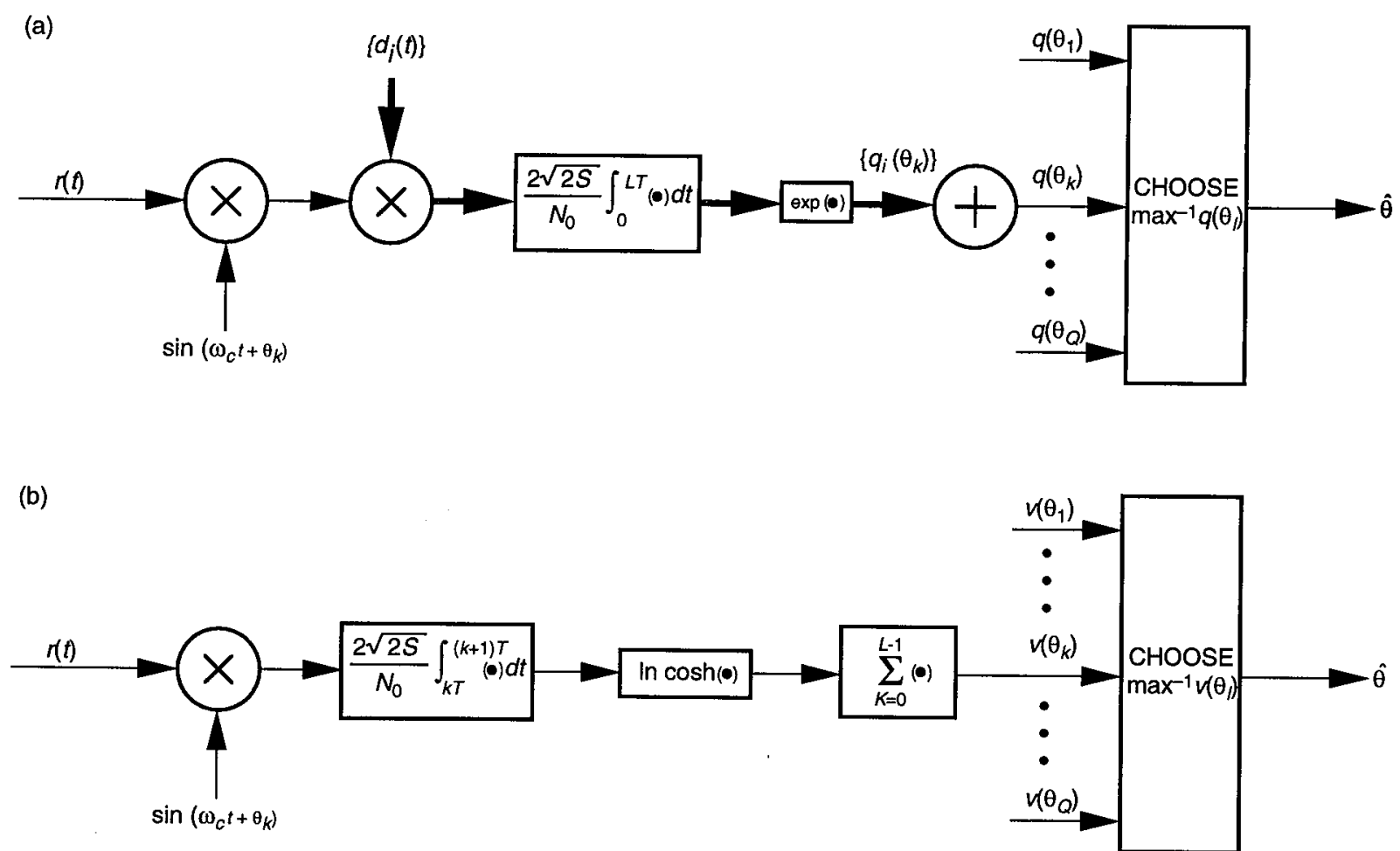

Fig. 2. Implementation of two AL open-loop phase estimators: (a) AL open-loop estimator no. 1-observation unpartitioned (quantized parallel implementation) and (b) AL open-loop estimator no. 3-observation partitioned (quantized parallel implementation).

Conceptually, a fourth optimum AL open-loop estimator, $\hat{\theta}_{A L_{4}}$, could be obtained by maximizing $\ln q_{A L}(\theta)$ with $q_{A L}(\theta)$ in its unpartitioned form. However, in view of the above discussion, $\hat{\theta}_{A L_{3}}$ and $\hat{\theta}_{A L_{4}}$ would be mathematically identical and, since $\hat{\theta}_{A L_{4}}$ appears to have no implementation advantage, we do not pursue it here. 
Closed-loop phase synchronization structures ${ }^{4}$ based on the four AL open-loop estimators are obtained by choosing as error signals, $e$, the functions respectively given by $d q_{A L}(\theta) / d \theta$ and $d \ln q_{A L}(\theta) / d \theta$ where $q_{A L}(\theta)$ and $\ln q_{A L}(\theta)$ each takes on its unpartitioned or partitioned form. For simplicity of notation, we shall refer to these four closed-loop structures as AL closed-loop nos. 1, 2, 3 and 4. The implementations corresponding to AL closed-loop no. 1 and AL closed-loop no. 3 (the two simplest implementations of the four) are illustrated in Figs. 3(a) and (b), the latter being what is commonly called an "I-Q MAP estimation loop" [8,9]. The special cases of Fig. 3(b), wherein the hyperbolic tangent nonlinearity is approximated by linear and hard limiter devices, corresponding respectively to low and high signal-tonoise ratio (SNR) conditions, are commonly called the "I-Q Costas loop" [4] and "I-Q polarity-type Costas loop" [5].
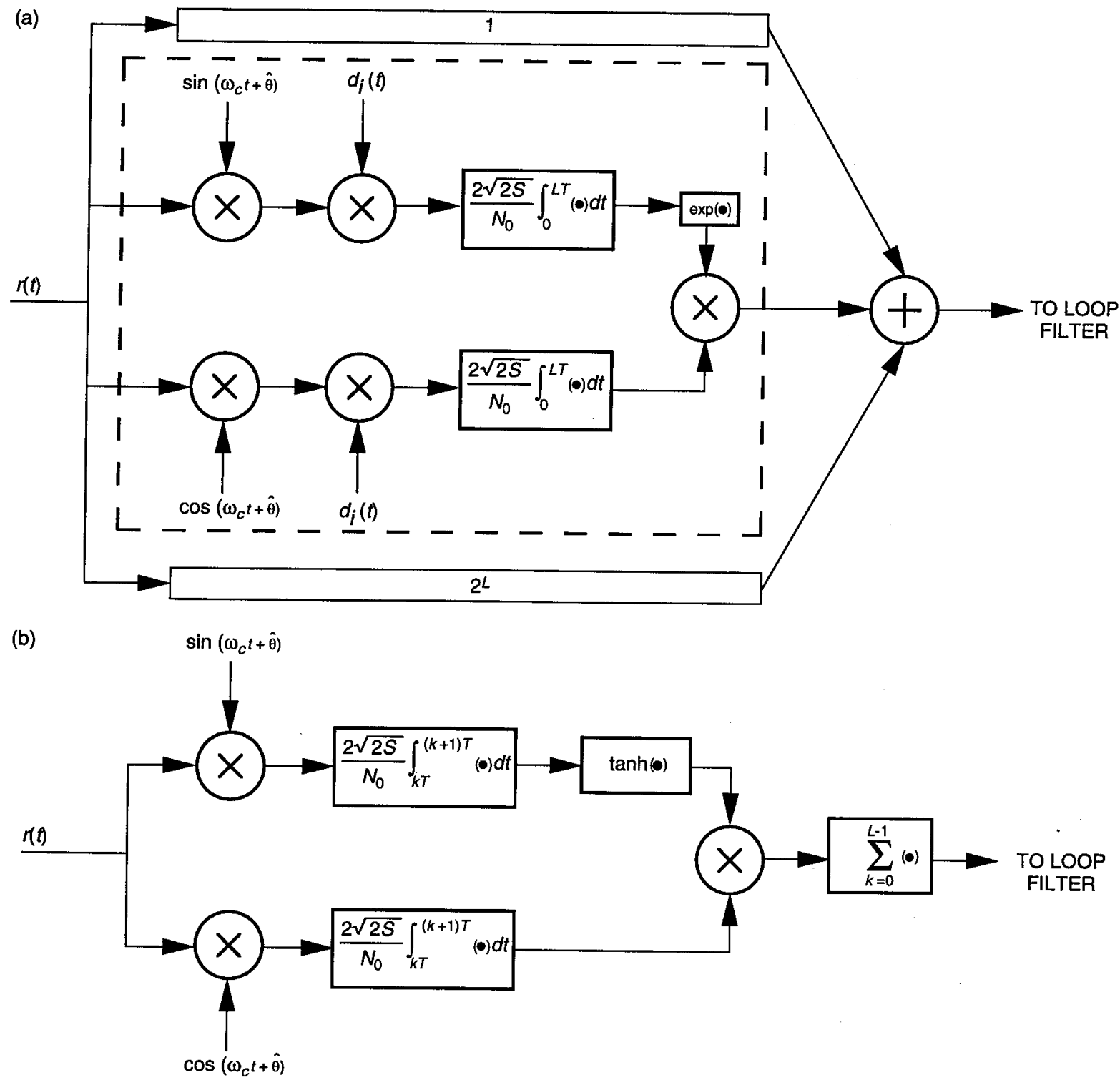

Fig. 3. Implementation of two AL closed loops: (a) AL closed loop no. 1-observation unpartitioned and (b) AL closed loop no. 3 -observation partitioned.

\footnotetext{
${ }^{4}$ For ease of illustration, we show only the portion of the closed loop that generates the loop error signal, which in the actual implementation becomes the input to the loop filter.
} 
Before proceeding, it is important to reemphasize that because of the monotonicity of the logarithm function, the $\mathrm{AL}$ open-loop phase estimates $\hat{\theta}_{A L_{3}}$ and $\hat{\theta}_{A L_{4}}$ are mathematically identical to $\hat{\theta}_{A L_{1}}$ and $\hat{\theta}_{A L_{2}}$ and thus yield identical performance. However, the equivalent statement is not necessarily true when considering the performances of the closed loops motivated by these four different AL formulations. More specifically, the closed loops motivated by $\hat{\theta}_{A L_{3}}$ and $\hat{\theta}_{A L_{4}}$ do not necessarily yield the same performance as those motivated by $\hat{\theta}_{A L_{1}}$ and $\hat{\theta}_{A L_{2}}$. The reason for this stems from the fact that the closed-loop performance (when properly normalized) is proportional to the derivative of $q_{A L}(\theta)$ (or $\ln q_{A L}(\theta)$ as appropriate) in the neighborhood of its maximum, which in general is different for $q_{A L}(\theta)$ and $\ln q_{A L}(\theta)$. However, we hasten to add that since partitioning does not change the functions $q_{A L}(\theta)$ or $\ln q_{A L}(\theta)$ themselves, the closed loops derived from either the partitioned or unpartitioned forms of the likelihood (or log likelihood) function should yield identical performance, i.e., AL closed-loop no. 1 and AL closedloop no. 2 will have identical performance, as will AL closed-loop no. 3 and AL closed-loop no. 4.

\section{B. Performance}

In assessing the performance of one closed-loop scheme versus another, one must be careful to normalize the loop parameters to allow a fair basis of comparison. In this article, the comparison will be made on the basis of mean-squared phase error, $\sigma_{\phi}^{2}$, for a fixed-loop bandwidth, $B_{L} .{ }^{5}$ This is the typical measure of performance used to describe a closed-loop phase synchronization structure when it is operating in its tracking mode.

An analysis of the closed-loop performance of AL closed-loop no. 1 [Fig. 3(a)] results in an expression for the mean-squared phase error given by ${ }^{6}$

$$
\sigma_{\phi}^{2}=\frac{1}{\rho}\left[\frac{L \sum_{i=1}^{2^{L}} \sum_{j=1}^{2^{L}} D_{i j} \exp \left\{2 R_{d}\left(L+D_{i}+D_{j}+D_{i j}\right)\right\}}{\left[\sum_{m=0}^{L}\left(\begin{array}{c}
L \\
m
\end{array}\right)(L-2 m) \exp \left\{R_{d}(3 L-4 m)\right\}\right]^{2}}\right] \triangleq \frac{1}{\rho \mathcal{S}_{L}}
$$

where

$$
\rho=\frac{S}{N_{0} B_{L}}, \quad R_{d}=\frac{S T}{N_{0}}
$$

and

$$
D_{i}=\sum_{k=0}^{L-1} d_{k} d_{i k}, \quad \sum_{k=0}^{L-1} d_{i k} d_{j k}
$$

with

$$
\begin{aligned}
\mathbf{d} \triangleq\left(d_{0}, d_{1}, \cdots, d_{L-1}\right) & =\text { transmitted data sequence } \\
\mathbf{d}_{i} \triangleq\left(d_{i 0}, d_{i 1}, \cdots, d_{i, L-1}\right) & =i \text { th data sequence; } \quad i=1,2, \cdots, 2^{L}
\end{aligned}
$$

\footnotetext{
${ }^{5}$ It is important at this point to emphasize that $B_{L}$, being proportional to the total loop gain, includes the slope of the loop S-curve at the origin as one of its factors. Since, in general, this slope is different for the various loops being investigated, it is absolutely essential to include this normalization (as we have done) in the definition of $B_{L}$ when comparing the performance of these loops.

${ }^{6}$ All of the performance results given in this article will be based upon the so-called "linear theory" [3], which assumes that the loop operates in a region of high loop SNR.
} 
In Eq. (9), $D_{i}$ represents the correlation of the $i$ th data sequence with the transmitted sequence, and $D_{i j}$ represents the correlation between the $i$ th and the $j$ th data sequences. Some properties of $D_{i}$ and $D_{i j}$ that are particularly useful in obtaining many of the results that follow are summarized as

$$
\begin{gathered}
\sum_{i=1}^{2^{L}} \sum_{j=1}^{2^{L}} D_{i j}=0 \\
\sum_{i=1}^{2^{L}} \sum_{j=1}^{2^{L}} D_{i} D_{i j}=\sum_{i=1}^{2^{L}} \sum_{j=1}^{2^{L}} D_{j} D_{i j}=0 \\
\sum_{i=1}^{2^{L}} \sum_{j=1}^{2^{L}} D_{i j}^{2}=2 L \sum_{m=0}^{L}\left(\begin{array}{c}
L \\
m
\end{array}\right)(L-2 m)^{2}=2^{2 L} L \\
\sum_{i=1}^{2^{L}} \sum_{j=1}^{2^{L}} D_{i} D_{j} D_{i j}=2^{2 L} L
\end{gathered}
$$

The factor $\mathcal{S}_{L}$ represents the loss of the effective loop SNR, $\rho^{\prime} \triangleq \sigma_{\phi}^{-2}$, relative to the loop SNR, $\rho$, of a phase-locked loop (PLL). For certain configurations, as we shall see, this loss is synonymous with what is commonly referred to as "squaring loss" $[4,10]$.

At first glance, it might appear that, for given values of $\rho, R_{d}$, and the observation length, $L$, the meansquared phase error would be a function of the particular sequence chosen as the transmitted sequence. It is easy to show that indeed this is not the case, i.e., $\sigma_{\phi}^{2}$ is independent of the sequence selected for $\mathrm{d}^{7}$ To see this, consider a sequence $\mathbf{d}_{l} \triangleq\left(d_{l 0}, d_{l 1}, \cdots, d_{l, L-1}\right) \neq \mathbf{d}$ and rewrite $D_{i}$ and $D_{i j}$ as

$$
\begin{gathered}
D_{i}=\sum_{k=0}^{L-1} d_{k} \underbrace{d_{l k} d_{l k}}_{=1} d_{i k}=\sum_{k=0}^{L-1} d_{k}^{\prime} d_{i k}^{\prime} \\
D_{i j}=\sum_{k=0}^{L-1} d_{i k} \underbrace{d_{l k} d_{l k}}_{=1} d_{j k}=\sum_{k=0}^{L-1} d_{i k}^{\prime} d_{j k}^{\prime}
\end{gathered}
$$

where $d_{k}^{\prime}=d_{k} d_{l k}$ represents the $k$ th element of some other possible transmitted sequence $\mathbf{d}^{\prime} \triangleq$ $\left(d_{0}^{\prime}, d_{1}^{\prime}, \cdots, d_{L-1}^{\prime}\right)$ and $d_{i k}^{\prime}=d_{l k} d_{i k}, d_{j k}^{\prime}=d_{l k} d_{j k}$ are the $k$ th elements of two other possible sequences $\mathbf{d}_{i}^{\prime} \triangleq\left(d_{i 0}^{\prime}, d_{i 1}^{\prime}, \cdots, d_{i, L-1}^{\prime}\right)$ and $\mathbf{d}_{j}^{\prime} \triangleq\left(d_{j 0}^{\prime}, d_{j 1}^{\prime}, \cdots, d_{j, L-1}^{\prime}\right)$, respectively. Since, in general, $\mathbf{d}^{\prime} \neq \mathbf{d}$ and since the summations on $i$ and $j$ in Eq. (7) range over all possible $\left(2^{L}\right)$ sequences, then substitution of Eq. (9) into Eq. (7) shows that $\sigma_{\phi}^{2}$ evaluated for a transmitted sequence equal to $\mathbf{d}^{\prime}$ is identical to that evaluated for a transmitted sequence equal to $d$.

Special cases of Eq. (7) corresponding to $L=1,2$, and 3 are given below:

\footnotetext{
${ }^{7}$ For convenience in the evaluation of Eq. (7), we may choose the all-1's sequence for d, in which case $D_{i}$ simplifies to $\sum_{k=0}^{L-1} d_{i k}$, which takes on values of $L-2 m, m=0,1,2, \cdots, L$.
} 


$$
\begin{aligned}
& \sigma_{\phi}^{2}=\frac{1}{\rho}\left[\frac{e^{8 R_{d}}-1}{\left(e^{3 R_{d}}-e^{-R_{d}}\right)^{2}}\right] ; \quad L=1 \\
& \sigma_{\phi}^{2}=\frac{1}{\rho}\left[\frac{e^{16 R_{d}}+2 e^{8 R_{d}}-3}{\left(e^{6 R_{d}}-e^{-2 R_{d}}\right)^{2}}\right] ; \quad L=2 \\
& \sigma_{\phi}^{2}=\frac{1}{\rho}\left[\frac{e^{24 R_{d}}+5 e^{16 R_{d}}+3 e^{8 R_{d}}-9}{\left(e^{9 R_{d}}+e^{5 R_{d}}-e^{R_{d}}-e^{-3 R_{d}}\right)^{2}}\right] ; \quad L=3
\end{aligned}
$$

Figure 4 is a plot of $\mathcal{S}_{L}$ (in $\mathrm{dB}$ ) versus $R_{d}$ (in $\mathrm{dB}$ ) corresponding to the three cases in Eq. (13). We observe that the performance of AL closed-loop no. 1 as implemented in Fig. 3(a) is clearly a function of the observation length of the corresponding open-loop estimator that motivated the structure.

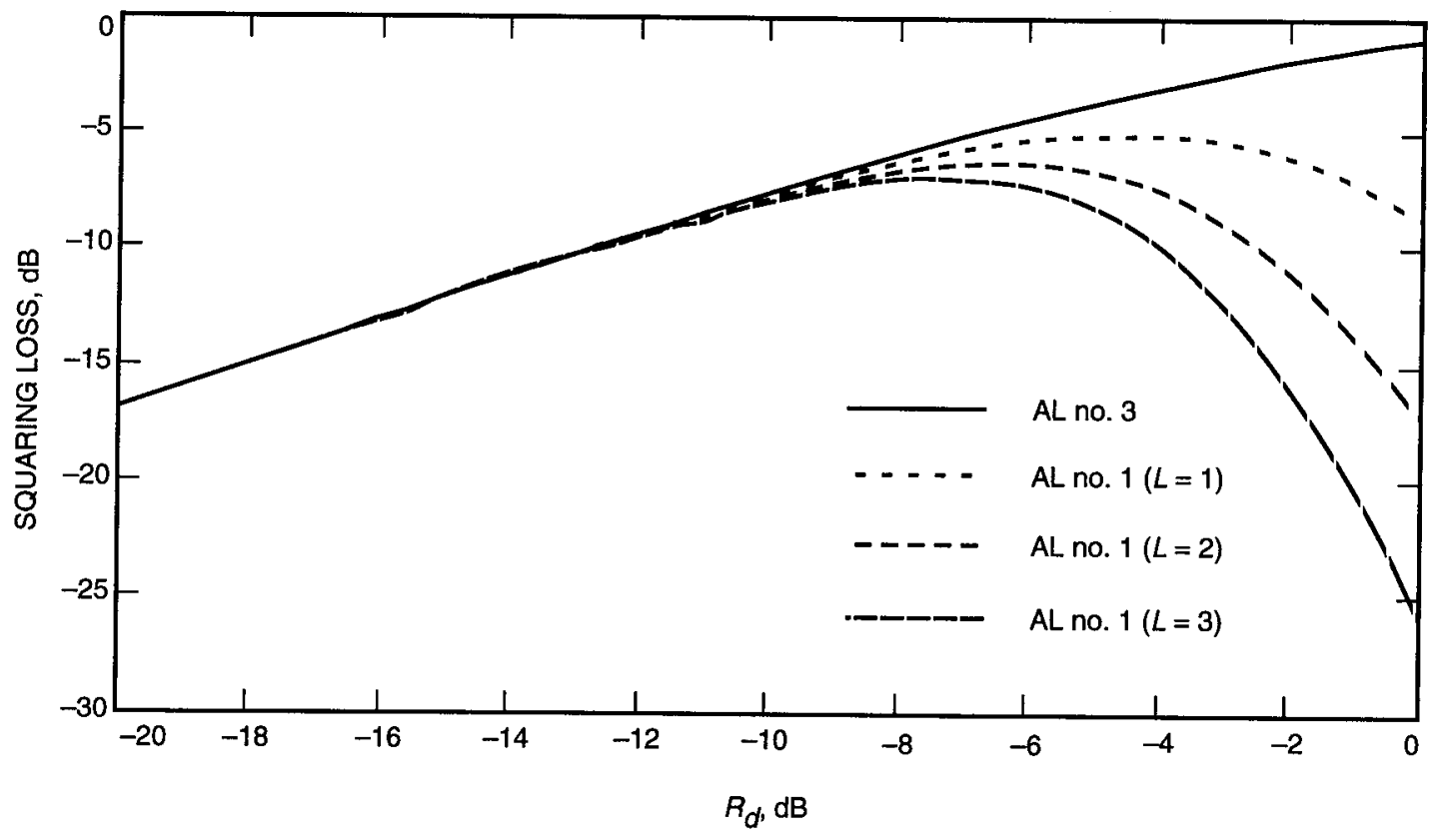

Fig. 4. Squaring-loss performance of AL closed-loop no. 1 with observation length $L$ as a parameter. I\&D weighting coefficients as determined by MAP estimation theory.

For large $R_{d}$, it is straightforward to show that $\sigma_{\phi}^{2}$ has the asymptotic behavior

$$
\sigma_{\phi}^{2} \cong \frac{1}{\rho} e^{2 L R_{d}} \rightarrow \mathcal{S}_{L} \cong e^{-2 L R_{d}}
$$

For small $R_{d}, \sigma_{\phi}^{2}$ has the asymptotic form

$$
\sigma_{\phi}^{2} \cong \frac{1}{\rho}\left(\frac{1}{2 R_{d}}\right) \rightarrow \mathcal{S}_{L} \cong 2 R_{d}
$$

which is independent of $L$. 
Looking at Eq. (14) and Fig. 4, one gets the impression (and rightfully so) that the mean-squared phase error of AL closed-loop no. 1 becomes unbounded as $R_{d} \rightarrow \infty$. This singular behavior can be traced to the fact that the $2 \sqrt{2 S} / N_{0}$ weighting coefficient of the two integrate-and-dump (I\&D) circuits in the closed loop of Fig. 3(a) becomes unbounded as $R_{d} \rightarrow \infty\left(N_{0} \rightarrow 0\right)$. Suppose instead that we were to replace this coefficient by an arbitrary constant, say $K_{0}$. From the standpoint of open-loop estimation of $\theta$, AL open-loop estimator no. 1 of Eq. (4) with $2 \sqrt{2 S} / N_{0}$ now replaced by $K_{0}$ would remain unchanged. That is, the choice of the weighting constant preceding the $L$-bit integration has no effect on the open-loop estimate. On the other hand, the choice of this weighting coefficient for the closed-loop scheme has a very definite bearing on its performance. In particular, with $2 \sqrt{2 S} / N_{0}$ replaced by $K_{0}$ in Fig. 3(a), the mean-squared phase error, previously given by Eq. (7), now becomes

$$
\sigma_{\phi}^{2}=\frac{1}{\rho}\left[\frac{L \sum_{i=1}^{2^{L}} \sum_{j=1}^{2^{L}} D_{i j} \exp \left\{K\left(D_{i}+D_{j}\right)+K^{2}\left(\frac{L}{2 R_{d}}\right)\left(1+\frac{D_{i j}}{L}\right)\right\}}{\left[\sum_{m=0}^{L}\left(\begin{array}{c}
L \\
m
\end{array}\right)(L-2 m) \exp \left\{K(L-2 m)+K^{2}\left(\frac{L}{4 R_{d}}\right)\right\}\right]^{2}}\right] \triangleq \frac{1}{\rho \mathcal{S}_{L}}
$$

where we have further normalized the weighting coefficient as $K \triangleq(\sqrt{S / 2}) K_{0} T$. Note that if we set $K_{0}=2 \sqrt{2 S} / N_{0}$ as before, then $K=2 R_{d}$ and Eq. (16) reduces to Eq. (7).

From Eq. (16), we see that as long as $K_{0}$ (or equivalently $K$ ) is finite (which would be the case in a practical implementation of the AL closed-loop scheme), the large SNR asymptotic behavior of $\mathrm{AL}$ closed-loop no. 1 now becomes

$$
\lim _{R_{d} \rightarrow \infty} \sigma_{\phi}^{2}=\lim _{N_{0} \rightarrow 0} \frac{N_{0} B_{L}}{S}\left[\frac{L \sum_{i=1}^{2^{L}} \sum_{j=1}^{2^{L}} D_{i j} \exp \left\{K\left(D_{i}+D_{j}\right)\right\}}{\left[\sum_{m=0}^{L}\left(\begin{array}{c}
L \\
m
\end{array}\right)(L-2 m) \exp \{K(L-2 m)\}\right]^{2}}\right]=0
$$

which is what one would expect. What is interesting is that, for any value of $R_{d}$, the value of $K$ that minimizes Eq. (16), which, from the standpoint of closed-loop performance as measured by mean-squared phase error, would be considered optimum, is $K \rightarrow 0$, independent of $R_{d}$. In fact, if one takes the limit of Eq. (16) as $K \rightarrow 0$ [this must be done carefully using the properties in Eq. (11)], the following result is obtained:

$$
\lim _{K \rightarrow 0} \sigma_{\phi}^{2} \triangleq\left(\sigma_{\phi}^{2}\right)_{\min }=\frac{1}{\rho}\left[1+\frac{1}{2 R_{d}}\right] \rightarrow\left(\mathcal{S}_{L}\right)_{\max }=\frac{1}{1+\left(1 / 2 R_{d}\right)}=\frac{2 R_{d}}{1+2 R_{d}}
$$

Interestingly enough, the result in Eq. (18), which is now independent of $L$, is also characteristic of the performance of the I-Q Costas loop [4], which is obtained as a low SNR approximation to AL closedloop no. 3. It is important to understand that the optimum closed-loop performance of Eq. (18) is a consequence of optimizing the weight (gain) $K$ for each value of $L$. If instead of doing this, one were to fix the gain $K$ for all values of $L$ (as suggested by the MAP estimation approach), the closed-loop performance (as measured by $\sigma_{\phi}^{2}$ with fixed-loop bandwidth) is suboptimum and indeed depends once again on $L$. One final note is that the small SNR behavior of Eq. (18) is identical to that of Eq. (15), the reason being that the value of $K=2 R_{d}$ used in arriving at Eq. (15) approaches the optimum value $(K=0)$ as $R_{d} \rightarrow 0$.

As previously stated, the performance of AL closed-loop no. 2 is identical to that of AL closed-loop no. 1 , and thus no further discussion is necessary. The performance of AL closed-loop no. 3 (and also 
AL closed-loop no. 4) has been obtained previously [8]. In particular, the mean-squared phase error performance of this loop is given by

$$
\sigma_{\phi}^{2}=\frac{1}{\rho}\left[\frac{\overline{\tanh ^{2}\left\{2 R_{d}-\sqrt{2 R_{d}} X\right\}}}{\left[\overline{\tanh \left\{2 R_{d}-\sqrt{2 R_{d}} X\right\}}\right]^{2}}\right] \triangleq \frac{1}{\rho \mathcal{S}_{L}}
$$

where $X$ is a zero-mean, unit-variance Gaussian random variable, and the over bar denotes statistical averaging over $X$. A plot of $\mathcal{S}_{L}$ versus $R_{d}$ is superimposed on the curves of Fig. 4 . We first note that the performance as given by Eq. (18) is independent of $L$. Furthermore, a comparison of the squaring loss as determined from Eq. (18) with that calculated from Eq. (17) reveals that the performance of AL closed-loop no. 3 is superior to that of AL closed-loop no. 1 with optimized gain for all values of $R_{d}$ (see Fig. 3 of [8]). As mentioned previously, if the hyperbolic tangent nonlinearity in Fig. 3(b) is approximated by a linear device (i.e., $\tanh x \cong x$ ), then the two loops have the same performance.

What is particularly interesting for AL closed-loop no. 3 is that even though the performance in Eq. (19) is computed assuming a weighting coefficient in front of the I\&Ds in Fig. 3 (b) equal to $2 \sqrt{2 S} / N_{0}$, the behavior of this loop is not singular in the limit as $R_{d} \rightarrow \infty$. Furthermore, it is natural to ask whether the above weighting coefficient is indeed optimum in the sense of minimizing $\sigma_{\phi}^{2}$. To answer this question, we proceed as we did for AL closed-loop no. 1, namely, we replace the weighting coefficient $2 \sqrt{2 S} / N_{0}$ by an arbitrary constant, say $K_{0}$, and proceed to optimize the performance with respect to the choice of this gain. ${ }^{8}$ Making this replacement produces a mean-squared phase error, analogous to Eq. (19), given by

$$
\sigma_{\phi}^{2}=\frac{1}{\rho}\left[\frac{\overline{\tanh ^{2}\left\{K\left[2 R_{d}-\sqrt{2 R_{d}} X\right]\right\}}}{\left[\overline{\tanh \left\{K\left[2 R_{d}-\sqrt{2 R_{d}} X\right]\right\}}\right]^{2}}\right] \triangleq \frac{1}{\rho \mathcal{S}_{L}}
$$

where, as before, we have further normalized the weighting coefficient as $K \triangleq K_{0} N_{0} / 2 \sqrt{2 S}$. Maximizing the squaring loss factor $\mathcal{S}_{L}$ (i.e., minimizing $\left.\sigma_{\phi}^{2}\right)$ in Eq. (20) results in $K=1\left(K_{0}=2 \sqrt{2 S} / N_{0}\right)$ for all values of $R_{d}$. Thus, for AL closed-loop no. 3, the optimum gain from the standpoint of closed-loop performance is precisely that dictated by the open-loop MAP estimation of $\theta$, and the best performance is that described by Eq. (19).

We conclude our discussion of AL closed loops by pointing out that, in view of the superiority of Eq. (19) over Eq. (18), AL closed-loop no. 3 outperforms AL closed-loop no. 1 for all values of $R_{d}$.

\section{Closed Loops Motivated by the ML Approach}

\section{A. Structures}

The ML approach to estimating the carrier phase, $\theta$, is to maximize (rather than to average) $p\left(r(t) \mid d_{i}(t), \theta\right)$ over all possible $\left(2^{L}\right)$ and equally likely data sequences. Analogous to AL open-loop estimator no. 1, "ML open-loop estimator no. 1" is defined by (see Fig. 1: $\hat{\theta}_{M L} \triangleq \max _{\theta}^{-1} q_{M L}(\theta)$, unpartitioned observation)

\footnotetext{
${ }^{8}$ Again we note that this replacement does not affect the open-loop estimation of $\theta$ using Eq. (6).
} 


$$
\begin{aligned}
& \hat{\theta}_{M L_{1}}=\max _{\theta}^{-1} q_{\hat{i}}(\theta) \\
& q_{\hat{i}}(\theta) \triangleq \exp \left(\max _{\left\{d_{i}(t)\right\}} \frac{2 \sqrt{2 S}}{N_{0}} \int_{0}^{L T} r(t) d_{i}(t) \sin \left(\omega_{c} t+\theta\right) d t\right)
\end{aligned}
$$

where $\hat{i}$ is the particular value of $i$ corresponding to the data waveform $d_{\hat{i}}(t)$ that achieves the maximization. Alternately, by breaking up the integration over the entire observation into a sum of integrals on each bit interval (the partitioned form of the observation) and recognizing that the data bits are iid binary random variables, then Eq. (21) evaluates to (see Fig. 1: $\hat{\theta}_{M L} \triangleq \max _{\theta}^{-1} q_{M L}(\theta)$, partitioned observation)

$$
\hat{\theta}_{M L_{2}}=\max _{\theta}^{-1} \prod_{k=0}^{L-1} \exp \left(\left|\frac{2 \sqrt{2 S}}{N_{0}} \int_{k T}^{(k+1) T} r(t) \sin \left(\omega_{c} t+\theta\right) d t\right|\right)
$$

This estimator is analogous to Eq. (5) and is called "ML open-loop estimator no. 2." Next, we obtain ML open-loop estimates by maximizing the natural logarithm of $q_{\hat{i}}(\theta)$. Using the product form of $q_{\hat{i}}(\theta)$ as in Eq. (22), one obtains (see Fig. 1: $\hat{\theta}_{M L} \triangleq \max _{\theta}^{-1} \ln q_{M L}(\theta)$, partitioned observation)

$$
\hat{\theta}_{M L_{3}}=\max _{\theta}{ }^{-1} \sum_{k=0}^{L-1}\left|\frac{2 \sqrt{2 S}}{N_{0}} \int_{k T}^{(k+1) T} r(t) \sin \left(\omega_{c} t+\theta\right) d t\right|
$$

which is analogous to Eq. (6) and therefore called the "ML open-loop estimator no. 3." Finally, we consider a fourth ML open-loop estimator, which is based on maximizing the natural logarithm of $q_{\hat{i}}(\theta)$ in its unpartitioned form of Eq. (15). This leads to "ML open-loop estimator no. 4," which is defined by (see Fig. 1: $\hat{\theta}_{M L} \triangleq \max _{\theta}^{-1} \ln q_{M L}(\theta)$, unpartitioned observation)

$$
\hat{\theta}_{M L_{4}}=\max _{\theta}-\frac{2 \sqrt{2 S}}{N_{0}} \int_{0}^{L T} r(t) d_{\hat{i}}(t) \sin \left(\omega_{c} t+\theta\right) d t
$$

Block diagram implementations of ML open-loop estimator no. 1 [Eq. (21)] and ML open-loop estimator no. 3 [Eq. (23)] are illustrated in Fig. 5 as representative of the four possibilities. In drawing these implementations, we have again quantized the unknown phase into $Q$ values and, thus, the maximization over the continuous phase parameter $\theta$ in Eqs. (21) and (23) is approximated by maximization over a $Q$-quantized version of this parameter.

As was true for the AL case, it is important to emphasize that the four ML open-loop phase estimates as described by Eqs. (21) through (24) are identical. However, we shall again see that this same statement is not true when considering the performances of the closed loops motivated by these four different ML formulations.

Closed-loop phase synchronization structures based on the four ML open-loop estimators are obtained as analogies of their $\mathrm{AL}$ counterparts, choosing as error signals, $e$, the derivatives with respect to $\theta$ of the functions being maximized in Eqs. (21) through (24), respectively. Analogous to the terminology used for the AL case, we shall refer to these four closed-loop structures as ML closed-loop nos. 1, 2, 3, 

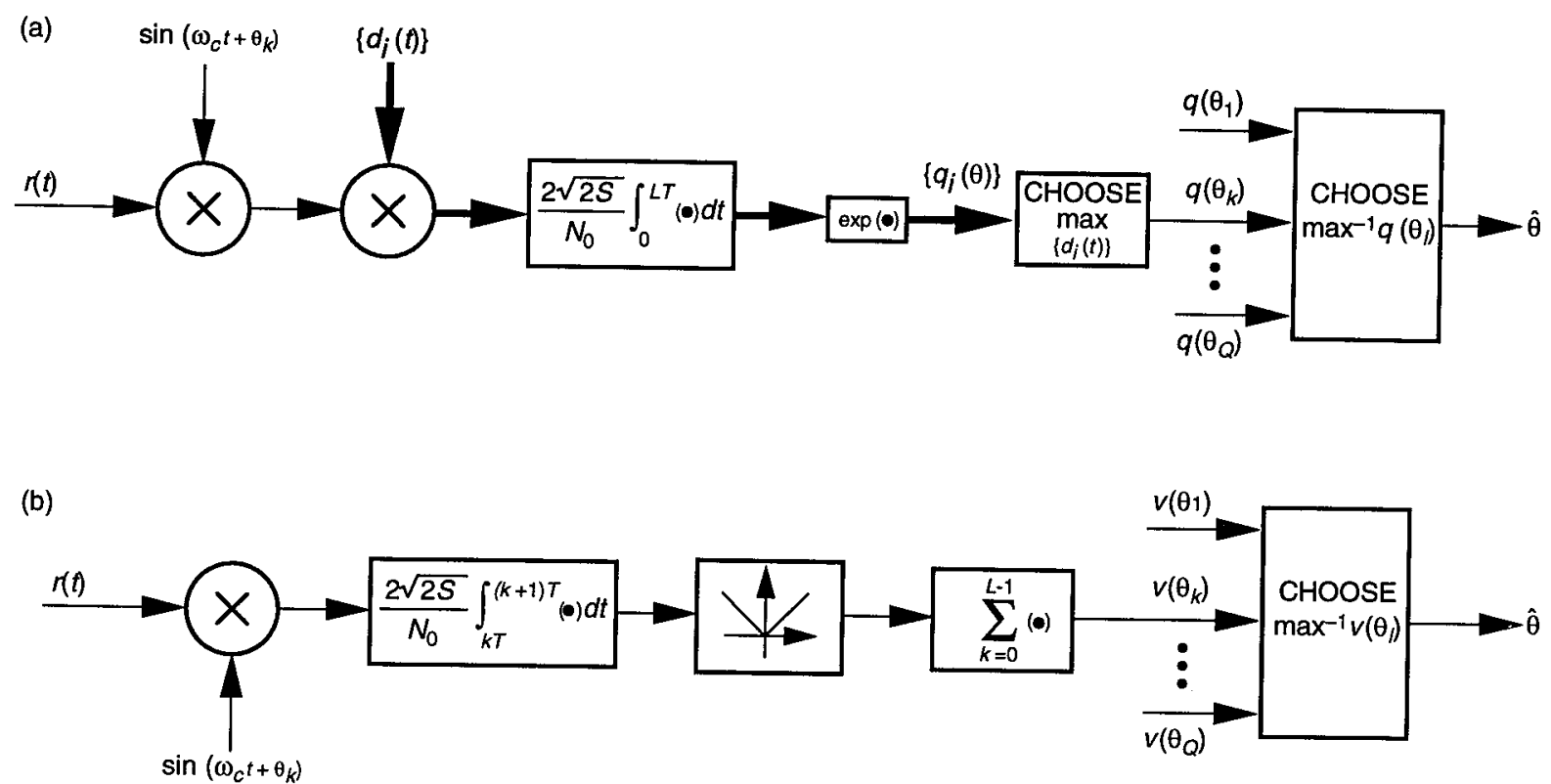

Fig. 5. Implementation of two ML open-loop phase estimators: (a) ML open-loop estimator no. 1-observation unpartitioned (quantized parallel implementation) and (b) ML open-loop estimator no. 3-observation partitioned
(quantized parallel implementation).

and 4. An implementation of ML closed-loop no. 1 is illustrated in Fig. 6(a). We also show here in Fig. 6(b) an implementation of ML closed-loop no. 1 (or ML closed-loop no. 2) for the special case of $L=1$ since, as we shall see shortly, this particular of $L$ yields the best performance. It is worthy of note that ML closed-loop no. 3 is identical in form to the I-Q polarity-type Costas loop [5], as can be seen in Fig. 6(c). (Note that the $L$-fold accumulator that precedes the loop filter can be omitted since it can be absorbed into the loop filter itself by renormalizing its bandwidth.) We recall that, in the AL case, the I-Q polarity-type Costas loop is obtained only as a high SNR approximation to closed-loop no. 3.

\section{B. Performance}

An analysis of the closed-loop performance of Fig. 6(a) results in an expression for the mean-squared phase error given by (see the Appendix for the derivation)

$$
\begin{aligned}
\sigma_{\phi}^{2} & =\frac{1}{\rho} e^{L K^{2} / 2 R_{d}} \frac{\left[\left(1-p_{2+}(0)\right) e^{2 K}+p_{2-}(0) e^{-2 K}\right]^{L}}{\left\{\left[\left(1-p_{+}(0)\right) e^{K}-p_{-}(0) e^{-K}\right]\left[\left(1-p_{+}(0)\right) e^{K}+p_{-}(0) e^{-K}\right]^{L-1}\right\}^{2}} \\
& \triangleq \frac{1}{\rho \mathcal{S}_{L}}
\end{aligned}
$$

where

$$
\begin{gathered}
p_{ \pm}(\phi) \triangleq \frac{1}{2} \operatorname{erfc}\left(\sqrt{R_{d}} \cos \phi \pm \frac{K}{2 \sqrt{R_{d}}}\right) \\
\left.p_{2 \pm}(\phi) \triangleq p_{ \pm}(\phi)\right|_{K \rightarrow 2 K}=\frac{1}{2} \operatorname{erfc}\left(\sqrt{R_{d}} \cos \phi \pm \frac{K}{\sqrt{R_{d}}}\right)
\end{gathered}
$$



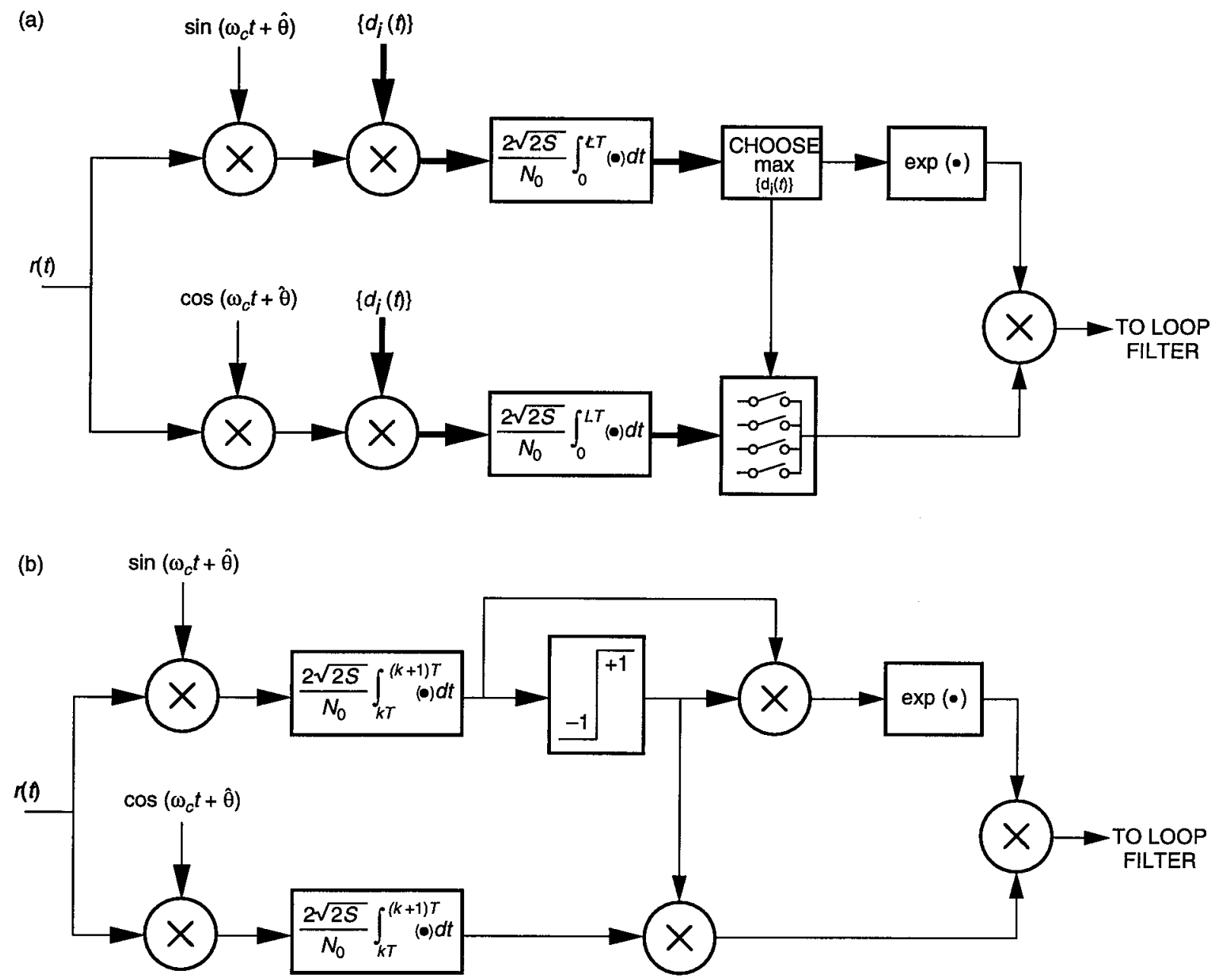

(c)

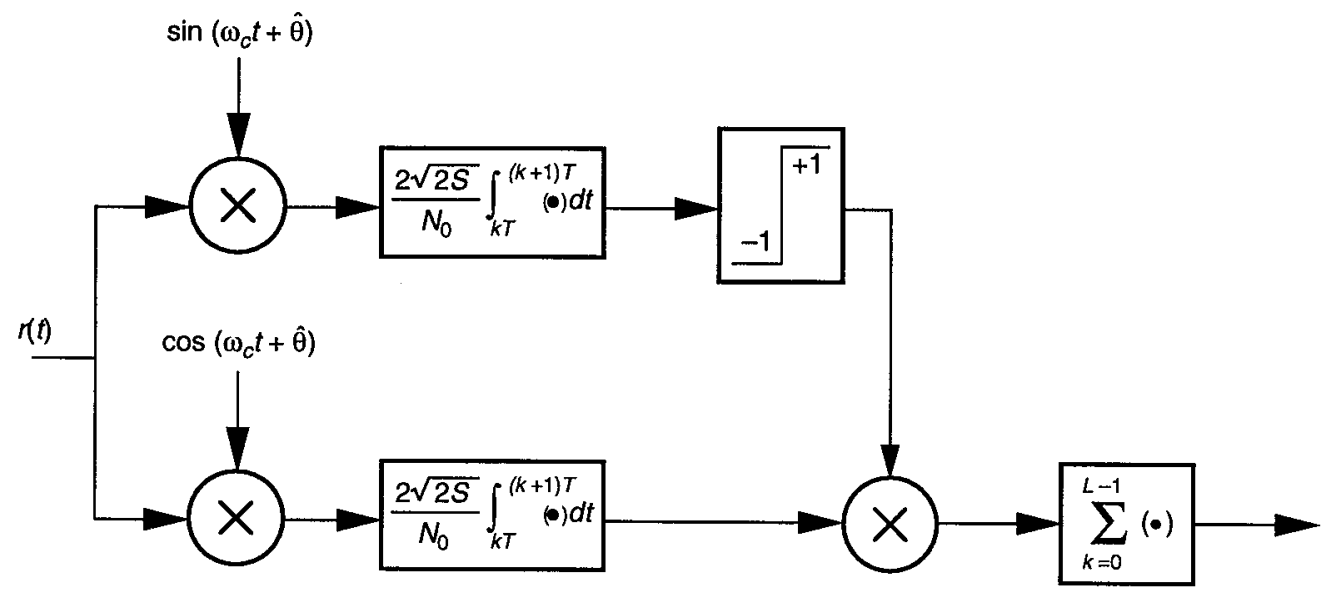

Fig. 6. Implementation of ML closed-loops: (a) ML closed-loop no. 1—observation unpartitioned, (b) ML closed-loop no. $2(L=1)$, and (c) ML closed-loop no. 3-observation partitioned. 
As we did for the analogous AL closed loop [see Fig. 3(a)], we have avoided the singular behavior of the mean-squared phase error as $R_{d} \rightarrow \infty$ by replacing the $2 \sqrt{2 S} / N_{0}$ coefficient in front of the I\&Ds in Fig. 6(a) by an arbitrary constant, say $K_{0}$, that remains finite as $N_{0} \rightarrow 0$ and further normalized the weighting coefficient as $K \triangleq(\sqrt{S / 2}) K_{0} T$. As long as $K_{0}$ (or equivalently $K$ ) is finite (which would be the case in a practical implementation of the ML closed-loop scheme), the large SNR asymptotic behavior of ML closed-loop no. 1 is

$$
\lim _{R_{d} \rightarrow \infty} \sigma_{\phi}^{2}=\lim _{N_{0} \rightarrow 0} \frac{N_{0} B_{L}}{S}=0
$$

as one would expect. What is indeed interesting is that, unlike the AL case, the value of $K$ that minimizes Eq. (25), which from the standpoint of closed-loop performance as measured by mean-squared phase error would be considered optimum, is not $K \rightarrow 0$. In fact, for each value of $R_{d}$ and $L$, there exists an optimum value of $K$ that unfortunately cannot be determined in closed form. Nevertheless, the optimum values of $K$ can be found numerically as a function of $R_{d}$ by maximizing $\mathcal{S}_{L}$ as determined from Eq. (25) for each value of $L$. The results are illustrated in Fig. 7. The corresponding values of $\left(\mathcal{S}_{L}\right)_{\max }$ are plotted versus $R_{d}$ in $\mathrm{dB}$ in Fig. 8 for the same values of $L$ as those in Fig. 7. Results obtained from a computer simulation of Fig. 6 (b) agree with these analytically obtained numerical results for $\left(\mathcal{S}_{L}\right)_{\max }$ within $0.1 \mathrm{~dB}$ at $R_{d}=-6 \mathrm{~dB}$.

From Fig. 8, we observe that the performance of ML closed-loop no. 1 becomes worse with increasing $L$, i.e., $L=1$ gives the best performance. Thus, the special case of the implementation in Fig. 6(a) corresponding to $L=1$, i.e., Fig. 6(b), is the configuration of most interest. Also in the limit as $L \rightarrow \infty$, the optimum value of $K$ approaches 0 independent of $R_{d}$. The corresponding value of $\mathcal{S}_{L}$ is determined by noting that for $K \rightarrow 0$ we have $p_{+}(0)=p_{-}(0)=p_{2+}(0)=p_{2_{-}}(0) \triangleq p=1 / 2$ erfc $\sqrt{R_{d}}$. Then from Eq. (25), we get

$$
\lim _{K \rightarrow 0} \sigma_{\phi}^{2}=\left(\sigma_{\phi}^{2}\right)_{0}=\frac{1}{\rho}(1-2 p)^{-2}=\frac{1}{\rho}\left(\operatorname{erfc}^{2} \sqrt{R_{d}}\right)^{-1} \rightarrow\left(\mathcal{S}_{L}\right)_{0}=\operatorname{erfc}^{2} \sqrt{R_{d}}
$$

which also is independent of the observation length $L$. Since the optimum value of $K$ is always greater than 0 (see Fig. 7), Eq. (28) also serves as a lower bound on the squaring-loss performance of ML closed-loop no. 1. Other reasons for including this limiting squaring-loss behavior in Fig. 8 will become apparent shortly when we consider the other ML closed-loop configurations.

As in the AL case, the performance of ML closed-loop no. 2 is identical to ML closed-loop no. 1 and needs no further discussion. Moving on to ML closed-loop no. 3, we previously identified this as being identical in form to the I-Q polarity-type Costas loop. Hence, its performance is independent of $L$ and is given by Eq. (28). Similarly, the performance of ML closed-loop no. 4 is also independent of $L$ and given by Eq. (28). Thus, we see that of the four ML closed loops, ML closed-loops nos. 1 and 2 are superior to ML closed-loops nos. 3 and 4 , which have performances that are identical and equal to those of the former in the worst case $(L \rightarrow \infty)$.

When the performance of the best ML closed-loop scheme (i.e., nos. 1 or 2) is compared with that of the best AL closed-loop scheme (i.e., nos. 3 or 4 ), we find that the latter, e.g., the I-Q MAP estimation loop, is superior to the former for all values of $R_{d}$. This comparison is illustrated in Fig. 9, where the squaring-loss performance of the two schemes is plotted versus $R_{d}$.

\section{Loop S-Curves}

It is of interest to examine the S-curve behavior of ML closed-loop no. 1 and compare it with that of ML closed-loop no. 3 and AL closed-loop no. 3. The equation describing the loop S-curve, $\eta(\phi)$, of ML 


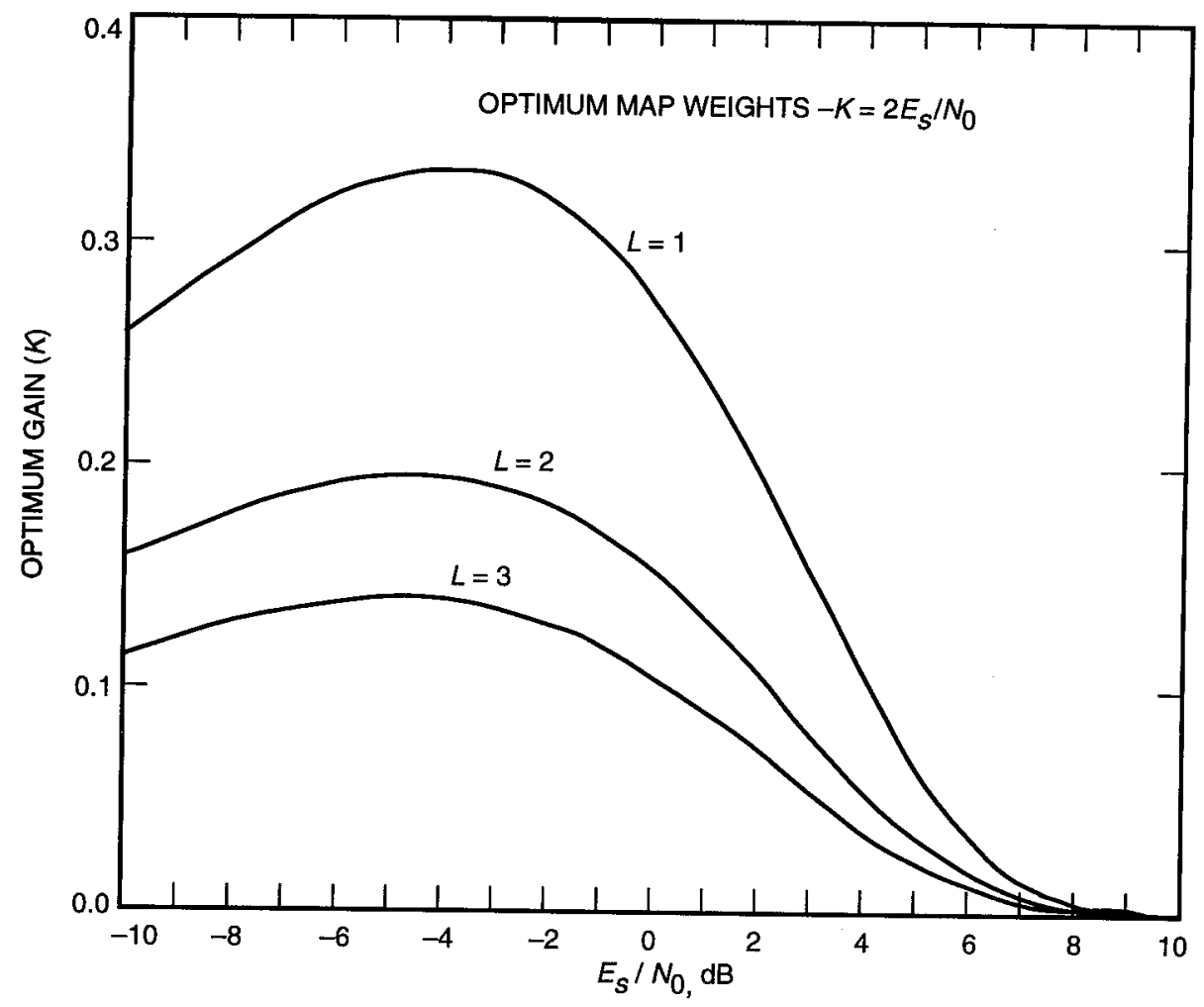

Fig. 7. Optimum weights (normalized) versus symbol SNR.

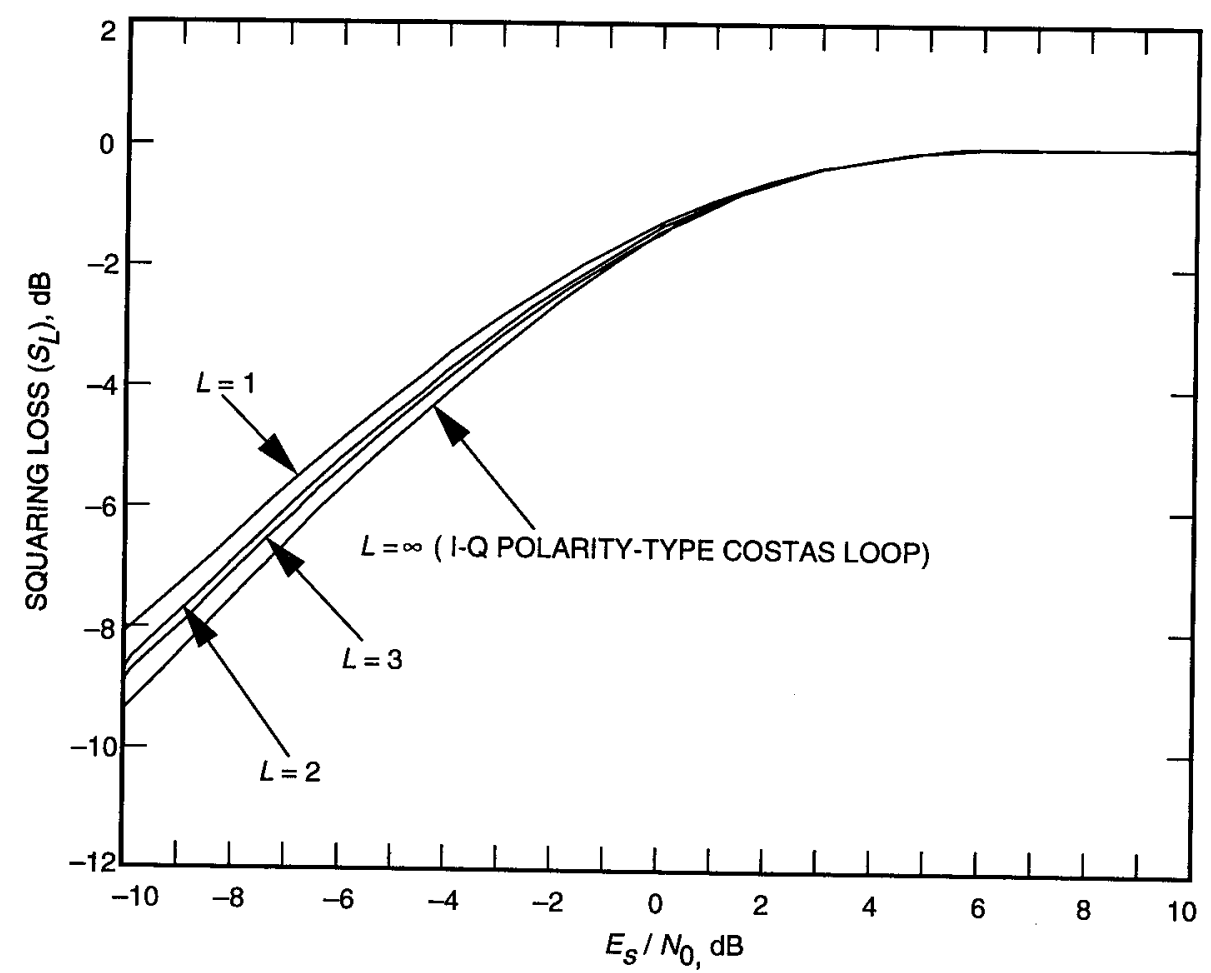

Fig. 8. Squaring loss versus symbol SNR for ML closed-loop no. 1 or no. 2. 
closed-loop no. 1 is derived in the Appendix as Eq. (A-9) with the special case of $L=1$ (already shown to yield the best tracking performance) given by Eq. (A-10). Figure 10 illustrates plots of $\eta(\phi)$ versus $\phi$ over one cycle of $\pi$ rad for $R_{d}=-5,2$, and $5 \mathrm{~dB}$, respectively, where in each case, $K$ has been chosen equal to the optimum value as determined from Fig. 7 . In the limit of small and large $R_{d}$, the S-curve approaches the following functional forms:

$$
\eta(\phi) \propto \begin{cases}\sin 2 \phi, & \text { small } R_{d} \\ \sin \phi \times \operatorname{sgn}(\cos \phi), & \text { large } R_{d}\end{cases}
$$

These limiting forms are identical to the same limiting behavior of the S-curves corresponding to ML closed-loop no. 3-the I-Q polarity-type Costas loop, and AL closed-loop no. 3-the I-Q MAP estimation loop.

\section{Conclusions}

Motivated by the theory of MAP carrier phase estimation, we have developed a number of closed-loop structures suitably derived from ML and AL functions. Several of these structures reduce to previously known closed-loop carrier phase synchronizers while others appear to be new. One of the new structures derived from ML considerations gives improved performance over the I-Q polarity-type Costas loop, which is also derived from these very same considerations. Of all the loops considered, however, the I-Q MAP estimation loop, which is derived from average log-likelihood considerations, is the best overall from a performance standpoint. We leave the reader with the thought that the structures proposed in this article are not exhaustive of the ways that closed-loop phase synchronizers can be derived from open-loop MAP estimation theory. Rather, they are given here primarily to indicate the variety of different closed-loop schemes that can be constructed simply from likelihood and log-likelihood functions.

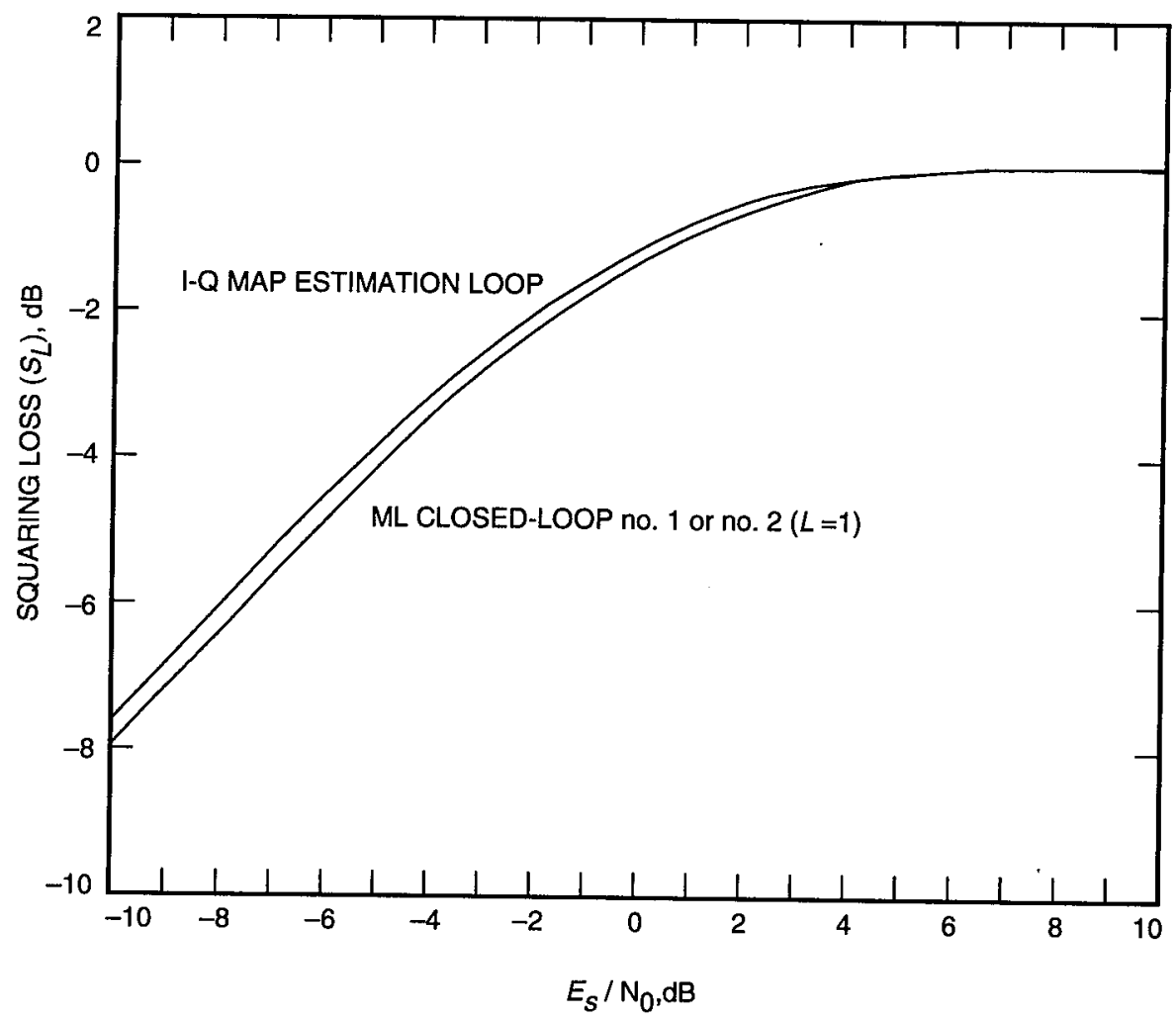

Fig. 9. A comparison of the squaring performances of $M L$ closed-loop no. 1 or no. 2 and the I-Q MAP estimation loop (AL closed loop no. 3). 


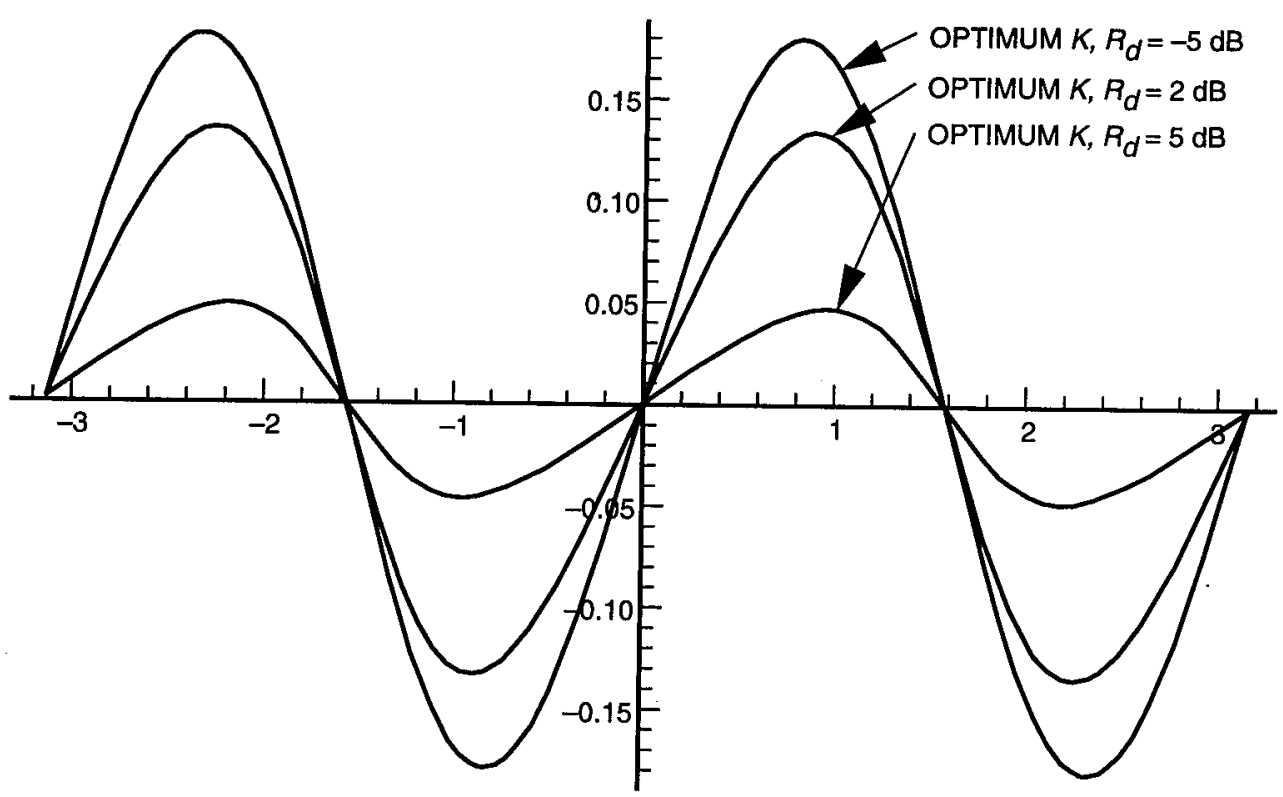

Fig. 10. Loop S-curves for ML closed-loop no. 1 or no. $2(L=1)$.

\section{References}

[1] C. W. Helstrom, Statistical Theory of Signal Detection, New York: Pergamon Press, 1960.

[2] H. L. VanTrees, Detection Estimation, and Modulation Theory, Part I, New York: John Wiley \& Sons, 1968.

[3] J. J. Stiffler, Theory of Synchronous Communications, Englewood Cliffs, New Jersey: Prentice Hall, Inc., 1971.

[4] W. C. Lindsey and M. K. Simon, "Optimum Design and Performance of Suppressed Carrier Receivers With Costas Loop Tracking," IEEE Transactions on Communications, vol. COM-25, no. 2, pp. 215-227, February 1977.

[5] M. K. Simon, "Tracking Performance of Costas Loops With Hard Limited InPhase Channel," IEEE Transactions on Communications, vol. COM-26, no. 4, pp. 420-432, April 1978.

[6] S. Dolinar, "A New Code for Galileo," The Telecommunications and Data Acquisition Progress Report 42-93, vol. January-March 1988, Jet Propulsion Laboratory, Pasadena, California, pp. 83-96, May 15, 1988.

[7] A. Mileant and S. Hinedi, "Overview of Arraying Techniques for Deep Space Communications," IEEE Transactions on Communications, vol. COM-42, nos. 2, 3, and 4, pp. 1856-1865, February, March, and April 1994.

[8] M. K. Simon, "On the Optimality of the MAP Estimation Loop for Tracking BPSK and QPSK Signals," IEEE Transactions on Communications, vol. COM-27, no. 1, pp. 158-165, January 1979. 
[9] M. K. Simon, "Optimum Receiver Structures for Phase-Multiplexed Modulations," IEEE Transactions on Communications, vol. COM-26, no. 6, pp. 865-872, June 1978.

[10] M. K. Simon, "On the Calculation of Squaring Loss in Costas Loops With Arbitrary Arm Filters," IEEE Transactions on Communications, vol. COM-26, no. 1, pp. 179-184, January 1978.

\section{Appendix}

\section{Derivation of the Closed-Loop Tracking Performance of ML Closed-Loop No. 1}

Consider the closed loop in Fig. 5(a), whose error signal, $e(t)$, at time $t=L T$ is characterized by

$$
e=\exp \left(K_{0} \int_{0}^{L T} r(t) d_{\hat{i}}(t) \sin \left(\omega_{c} t+\hat{\theta}\right) d t\right) \times K_{0} \int_{0}^{L T} r(t) d_{\hat{i}}(t) \cos \left(\omega_{c} t+\hat{\theta}\right) d t
$$

Substituting $r(t)$ of Eq. (1) into Eq. (A-1) results in

$$
\begin{aligned}
e= & \exp \left\{K_{0} \sqrt{\frac{S}{2}}\left(\int_{0}^{L T} d(t) d_{\hat{i}}(t) d t\right) \cos \phi+K_{0} \int_{0}^{L T} n(t) d_{\hat{i}}(t) \sin \left(\omega_{c} t+\hat{\theta}\right) d t\right\} \\
& \times\left[K_{0} \sqrt{\frac{S}{2}}\left(\int_{0}^{L T} d(t) d_{\hat{i}}(t) d t\right) \sin \phi+K_{0} \int_{0}^{L T} n(t) d_{\hat{i}}(t) \cos \left(\omega_{c} t+\hat{\theta}\right) d t\right]
\end{aligned}
$$

In view of the rectangular phase shape assumed in Eq. (2) for the transmitted data waveform, $d(t)$, Eq. (A-2) can be written in the discrete form

$$
e=\exp \left\{K_{0} \sum_{k=0}^{L-1} d_{\hat{i} k}\left(\sqrt{\frac{S}{2}} d_{k} \cos \phi+n_{s k}\right)\right\} \times\left[K_{0} \sum_{k=0}^{L-1} d_{\hat{\imath} k}\left(\sqrt{\frac{S}{2}} d_{k} \sin \phi+n_{c k}\right)\right]
$$

where

$$
n_{s k} \triangleq \int_{k T}^{(k+1) T} n(t) \sin \left(\omega_{c} t+\hat{\theta}\right) d t ; \quad n_{c k} \triangleq \int_{k T}^{(k+1) T} n(t) \cos \left(\omega_{c} t+\hat{\theta}\right) d t
$$

are zero mean iid Gaussian random variables with variance $\sigma_{n_{c k}}^{2}=\sigma_{n_{s k}}^{2}=N_{0} T / 4$ and $d_{\hat{i} k} \triangleq$ $\operatorname{sgn}\left(\sqrt{S / 2} d_{k} \cos \phi+n_{s k}\right)$. Introducing the further normalization $K=K_{0} T \sqrt{S / 2}$ (note that when $K_{0}=2 \sqrt{2 S} / N_{0}$, i.e., the gain suggested by the open-loop MAP estimation theory, then $K=2 R_{d}$ ) and 
normalizing $n_{s k}$ and $n_{c k}$ to unit variance Gaussian random variables, $N_{s k}$ and $N_{c k}$, respectively, Eq. (A-4) becomes

$$
e=\exp \left\{K \sum_{k=0}^{L-1} d_{\hat{i} k}\left(d_{k} \cos \phi+\frac{1}{\sqrt{2 R_{d}}} N_{s k}\right)\right\} \times\left[K \sum_{k=0}^{L-1} d_{\hat{i} k}\left(d_{k} \sin \phi+\frac{1}{\sqrt{2 R_{d}}} N_{c k}\right)\right]
$$

with $d_{\hat{i} k} \triangleq \operatorname{sgn}\left(d_{k} \cos \phi+\left(1 / \sqrt{2 \pi R_{d}}\right) N_{s k}\right)$.

Let $\eta(\phi)$ denote the signal component (mean) of the error sample $e$. Then, because of the independence of the $N_{s k}$ 's and $N_{c k}$ 's, we have

$$
\begin{aligned}
\eta(\phi)= & K \sin \phi\left(\sum_{k=0}^{L-1} \overline{\left.d_{\hat{i} k} d_{k} \exp \left\{K d_{\hat{i} k}\left(d_{k} \cos \phi+\frac{1}{\sqrt{2 R_{d}}} N_{s k}\right)\right\}^{N_{s k}}\right)}\right. \\
& \times \prod_{\substack{l=0 \\
l \neq k}}^{L-1} \overline{\exp \left\{K d_{\hat{\imath} l}\left(d_{l} \cos \phi+\frac{1}{\sqrt{2 R_{d}}} N_{s l}\right)\right\}}{ }^{N_{s l}}
\end{aligned}
$$

where the over bar denotes statistical averaging. It is straightforward to show that the statistical averages required in Eq. (A-6) are independent of the data bits. That is,

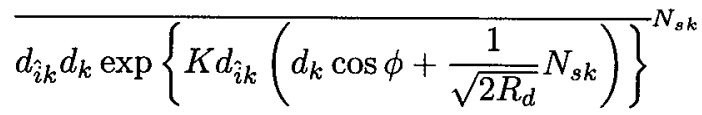

is independent of whether $d_{k}=1$ or $d_{k}=-1$ and

$$
\left.\overline{\exp \left\{K d_{\hat{i} l}\left(d_{l} \cos \phi+\frac{1}{\sqrt{2 R_{d}}} N_{s l}\right)\right\}}\right\}^{N_{s l}}
$$

is independent of whether $d_{l}=1$ or $d_{l}=-1$. Performing these statistical averages gives the closed form results

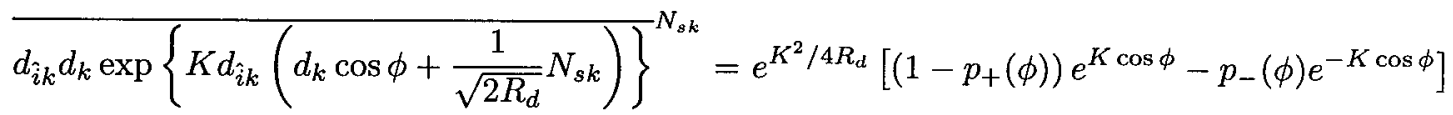

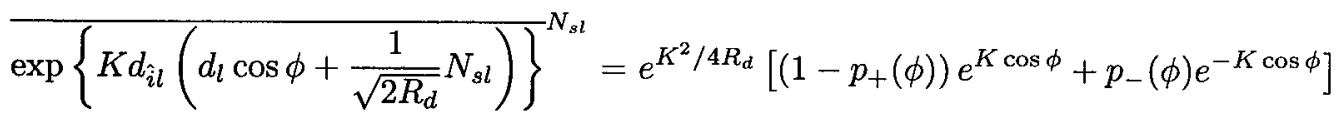

where 


$$
p_{ \pm}(\phi)=\frac{1}{2} \operatorname{erfc}\left(\sqrt{R_{d}} \cos \phi \pm \frac{K}{2 \sqrt{R_{d}}}\right)
$$

Finally, since Eq. (A-7a) is independent of $k$ and Eq. (A-7b) is independent of $l$, then substituting these results into Eq. (A-6), we get

$$
\begin{aligned}
\eta(\phi)= & (K \sin \phi) L e^{L K^{2} / 4 R_{d}}\left[\left(1-p_{+}(\phi)\right) e^{K \cos \phi}-p_{-}(\phi) e^{-K \cos \phi}\right] \\
& \times\left[\left(1-p_{+}(\phi)\right) e^{K \cos \phi}+p_{-}(\phi) e^{-K \cos \phi}\right]^{L-1}
\end{aligned}
$$

which represents the S-curve of the loop. For $L=1$, Eq. (A-9) simplifies to

$$
\eta(\phi)=(K \sin \phi) e^{K^{2} / 4 R_{d}}\left[\left(1-p_{+}(\phi)\right) e^{K \cos \phi}-p_{-}(\phi) e^{-K \cos \phi}\right]
$$

which, using the definition of $p_{ \pm}$, is periodic in $\phi$ with period $\pi$.

The slope of the S-curve at $\phi=0$ is needed for computing the closed-loop mean-squared phase error performance. Differentiating Eq. (A-10) with respect to $\phi$ and evaluating the result at $\phi=0$ gives

$$
\begin{aligned}
\left.K_{\eta} \triangleq \frac{d \eta(\phi)}{d \phi}\right|_{\phi=0}= & K L e^{L K^{2} / 4 R_{d}}\left[\left(1-p_{+}(0)\right) e^{K \cos \phi}-p_{-}(0) e^{-K \cos \phi}\right] \\
& \times\left[\left(1-p_{+}(0)\right) e^{K \cos \phi}+p_{-}(0) e^{-K \cos \phi}\right]^{L-1}
\end{aligned}
$$

The noise component of $e$ evaluated at $\phi=0$ is

$$
N=\exp \left\{K \sum_{k=0}^{L-1} d_{\hat{\imath} k}\left(d_{k} \cos \phi+\frac{1}{\sqrt{2 R_{d}}} N_{s k}\right)\right\} \times\left[K \sum_{k=0}^{L-1} d_{\hat{i} k} \frac{1}{\sqrt{2 R_{d}}} N_{c k}\right]
$$

Which is zero mean and has variance

$$
\sigma_{N}^{2}=\overline{\exp \left\{2 K \sum_{k=0}^{L-1} d_{\hat{i} k}\left(d_{k}+\frac{1}{\sqrt{2 R_{d}}} N_{s k}\right)\right\} \times\left[K^{2}\left(\sum_{k=0}^{L-1} d_{\hat{i} k} \frac{1}{\sqrt{2 R_{d}}} N_{c k}\right)^{2}\right]}
$$

Averaging first over the $N_{c k}$ 's, we get

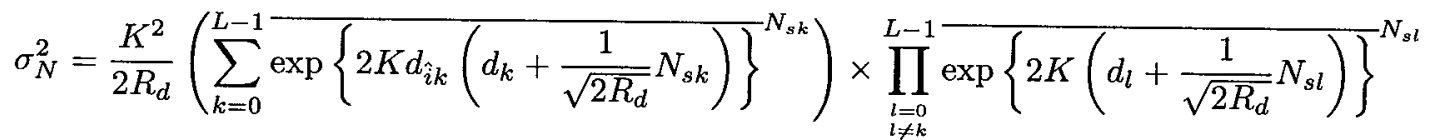

Using Eq. (A-7) to evaluate the averages over the $N_{s k}$ 's, we get 


$$
\sigma_{N}^{2}=\frac{K^{2} L}{2 R_{d}} e^{L K^{2} / R_{d}}\left[\left(1-p_{2+}(0)\right) e^{2 K}+p_{2-}(0) e^{-2 K}\right]^{L}
$$

where

$$
\left.p_{2 \pm}(\phi) \triangleq p_{ \pm}(\phi)\right|_{K \rightarrow 2 K}=\frac{1}{2} \operatorname{erfc}\left(\sqrt{R_{d}} \cos \phi \pm \frac{K}{\sqrt{R_{d}}}\right)
$$

Since $e(t)$ is a piecewise constant (over intervals of length $L T$ ) random process with independent increments, its statistical autocorrelation function is triangular and given by

$$
R_{e}(\tau) \triangleq\langle E\{e(t) e(t+\tau)\}\rangle=\left\{\begin{array}{cc}
\sigma_{N}^{2}, & |\tau| \leq L T \\
0, & \text { otherwise }
\end{array}\right\}
$$

Where $\langle\bullet\rangle$ denotes time averaging, which is necessary because of the cyclostationarity of $e(t)$. As is customary in analyses of this type, we assume a narrow-band loop, i.e., a loop bandwidth $B_{L} \ll 1 / T$. Then, $e(t)$ is approximated as a delta-correlated process with effective power spectral density:

$$
\frac{N_{0}^{\prime}}{2} \triangleq \int_{-\infty}^{\infty} R_{e}(\tau) d \tau=L T \sigma_{N}^{2}
$$

Finally, the mean-squared phase error for the closed loop is

$$
\sigma_{\phi}^{2}=\frac{N_{0}^{\prime} B_{L}}{K_{\eta}^{2}}
$$

which, with substitution of Eqs. (A-11) and (A-18), results in Eq. (26) of the main text. 